\title{
AVALIAÇÃO DA PERFORMANCE MASTIGATÓRIA EM INDIVÍDUOS RESPIRADORES NASAIS E ORAIS
}

\author{
Masticatory performance evaluation in patients \\ with nasal and mouth breathing
}

Rosany Larissa Brito de Oliveira (1), Walter Pinheiro Noronha ${ }^{(2)}$, Leonardo Rigoldi Bonjardim (3)

\section{RESUMO}

Objetivo: avaliar, por meio da mastigação sequencial de uma "pastilha" artificial de Optosil, o desempenho mastigatório de indivíduos respiradores nasais e orais. Método: participaram sessenta sujeitos dentados na faixa etária de 14 a 22 anos. Uma "pastilha" de Optosil foi fornecida a cada participante, que executou 20 ciclos mastigatórios, os fragmentos foram colocados numa coluna de sete peneiras, sendo, posteriormente pesados numa balança de precisão. Os dados obtidos foram submetidos à análise descritiva e inferencial (Teste T e ANOVA). Resultados: os grupos respiradores nasais e respiradores orais foram constituídos de 30 jovens cada, com idade média de 17,87 e 17,83 anos respectivamente. Com relação ao gênero houve predominância do gênero feminino no grupo respirador oral e do gênero masculino no grupo respirador nasal Os cinco diagnósticos mais prevalentes no grupo respirador oral foram hipertrofia de adenóide, rinite alérgica, sinusite, asma e hipertrofia de amígdala. Por fim, ao ser realizado o Teste $\mathrm{T}$, não foi encontrada diferença estatística na performance mastigatória nos respiradores orais e nasais $(p>0,05)$. Conclusão: o padrão respiratório não foi um fator determinante na performance mastigatória.

DESCRITORES: Mastigação; Eficiência; Respiração Bucal

\section{INTRODUÇÃO}

A mastigação é a função mais importante do sistema estomatognático, pois com ela se inicia o processo digestivo, tendo como objetivo a degradação mecânica dos alimentos, reduzindo-os a um tamanho adequado para a deglutição, estimulando também, o crescimento, desenvolvimento e manutenção da saúde do sistema estomatognático'.

A performance mastigatória, é a mensuração da capacidade do indivíduo de fragmentar o alimento.

(1) Cirurgiã-dentista; Graduada em Odontologia pela Universidade Federal de Sergipe - UFS. Aracaju, Sergipe, Brasil.

(2) Cirurgião-dentista; Professor Efetivo do Departamento de Odontologia, Universidade Federal de Sergipe - UFS. Aracaju, Sergipe, Brasil. Mestre em Ortodontia Bioprogressiva pela São Leopoldo Mandick, Campinas, São Paulo, Brasil.

(3) Cirurgião-dentista; Professor Efetivo do Departamento de Fisiologia, Universidade Federal de Sergipe - UFS. Aracaju, Sergipe, Brasil. Doutor em Odontologia pela Universidade Estadual de Campinas - UNICAMP. Campinas, São Paulo, Brasil.

Conflito de interesses: inexistente
Em geral essa mensuração é feita pela tomada de uma amostra de alimento expelida por um indivíduo depois de tê-lo mastigado.

A síndrome do respirador oral, por sua vez, é o conjunto de sinais e sintomas de quem respira total ou parcialmente pela boca $^{2}$, podendo estar ou não, associada à obstrução nasal ${ }^{3}$. Somente as cavidades nasais possuem condições perfeitas para que o ar chegue aos pulmões na temperatura ideal, favorecendo uma excelente oxigenação ao organismo e proporcionando uma melhor qualidade de vida ao indivíduo 4 .

Moyers ${ }^{5}$ acredita que a obstrução nasal provoca alterações na postura de língua, lábios e mandíbula, ou seja, os tecidos moles em desequilíbrio ocasionaram mudanças na morfologia craniofacial e maloclusão. Crianças respiradoras orais têm uma tendência a ter uma mandíbula retraída e uma maior inclinação dos incisivos superiores, além disso, o espaço aéreo nasofaríngeo e posterior são grandemente reduzidos ${ }^{6-8}$. De tal modo, o respirador oral não se alimenta bem, prejudicando seu desenvolvimento como um todo ${ }^{9,10}$. 
O objetivo do presente estudo foi avaliar, por meio da mastigação sequencial de uma "pastilha" artificial de Optosil, o desempenho mastigatório de indivíduos respiradores nasais e orais na dentição permanente. Ambos foram ainda classificados quanto ao padrão oclusal em Classe I, Classe II e Classe III de Angle.

\section{MÉTODO}

\section{Amostra}

Esta pesquisa trata-se de um estudo descritivo transversal. A amostra foi constituída de 60 sujeitos na faixa etária de 14 a 22 anos de idade atendidos no Hospital Universitário da Universidade Federal de Sergipe e no Centro de Especialidades Médicas (CEMAR) em Aracaju-SE. Os sujeitos foram distribuídos em dois grupos: Grupo RO (respiradores orais), composto de 30 participantes, sendo 10 classe I de Angle, 10 Classe II de Angle e 10 Classe III de Angle; Grupo RN (respiradores nasais), composto por 30 sujeitos, sendo destes 10 Classe I de Angle, 10 Classe II de Angle e 10 Classe III de Angle. Foi critério de inclusão nesse estudo a presença dos elementos dentários permanentes no arco, sendo facultativa a presença dos terceiro molares, sendo excluídos aqueles sujeitos com distúrbios cognitivos, motores e doenças sistêmicas que impossibilitassem a realização dos exames.

Para fazer parte do estudo, antes os participantes e/ou seus responsáveis, quando menor de idade, assinaram o Termo de Consentimento Livre e Esclarecido.

\section{Avaliação do padrão respiratório}

Os indivíduos respiradores orais e nasais foram diagnosticados através do uso da placa oronasal, conforme preconizado por Barreto ${ }^{11} \mathrm{em}$ um estudo realizado em 2003. A placa oronasal é feita em aço inoxidável, apresentando um tamanho de $10 \mathrm{~cm}$ de comprimento por $5 \mathrm{~cm}$ de largura, dobrada ao meio no sentido do seu comprimento formando assim, um ângulo de $90^{\circ}$.

A placa foi colocada logo abaixo das narinas, de forma a ficar com o ângulo de $90^{\circ}$ com um lado apoiado no filtro labial e o outro na columela do nariz e de acordo com o fluxo respiratório poderá haver o embaçamento de uma das faces da placa. Assim, o modo respiratório foi classificado em respiração nasal quando ocorreu o embaçamento da superfície da placa voltada para o nariz, respiração oronasal quando houve embaçamento de ambas as superfícies e respiração oral se ocorreu embaçamento da superfície da placa voltada para a boca.

\section{Patologias respiratórias encontradas no respirador oral}

Após a seleção dos respiradores orais, seus prontuários foram consultados para verificar quais patologias respiratórias estavam presentes nesse grupo. Só foram aceitos os prontuários que apresentavam o exame otorrinolaringológico clínico completo.

\section{Avaliação da classe oclusal de Angle}

Foi realizado o exame intrabucal com espátula de madeira, luvas, máscara e gorro, para que se pudesse observar e detectar, em máxima intercuspidação habitual, a presença da Classe I, II ou III seguindo o protocolo proposto por Angle apud Araújo12.

- Classe I: A cúspide mésio-vestibular do primeiro molar superior oclui no sulco central do primeiro molar inferior.

- Classe II: A cúspide mésio-vestibular do primeiro molar superior oclui com a vertente distal do segundo pré-molar inferior.

- Classe III: Caracteriza-se por apresentar o primeiro molar inferior em posição mesial na relação com o primeiro molar superior.

\section{Avaliação da performance mastigatória}

Para a avaliação da performance mastigatória foram utilizadas "pastilhas" do material teste Optosil. O Optosil foi manipulado de acordo com as orientações do fabricante. A massa formada foi colocada em um molde constituído de uma placa de alumínio com orifícios de $2 \mathrm{~cm}$ de diâmetro por 0,5 $\mathrm{cm}$ de altura. O Optosil foi pressionado nas perfurações (pressão hidráulica de 25 libras $/ \mathrm{pol}^{2}$ ) por 10 minutos, entre duas placas de vidro cobertas com folha de teflon. Após, as amostras foram pesadas numa balança analítica digital de precisão, e quando houve variação no peso, os tabletes foram recortados manualmente até que todos não variassem mais que $0,05 \mathrm{~g}$, conforme se pode observar na Figura 1.

Os voluntários foram devidamente treinados no que diz respeito aos movimentos mastigatórios, antes da execução do teste propriamente dito. Foi entregue a cada voluntário um tablete de Optosil, e este mastigou o tablete durante 20 ciclos mastigatórios. As partículas recolhidas foram lavadas com água e sabão neutro apropriados, sendo posteriormente colocadas numa série de 07 peneiras (tamises) com aberturas de $5,6 \mathrm{~mm}$ diminuindo até $0,71 \mathrm{~mm}$ e, este conjunto de peneiras levado a um agitador eletromagnético de tamises (Bertel Ltda) por 5 minutos. As partículas retidas em cada peneira foram pesadas novamente em balança analítica de precisão. 

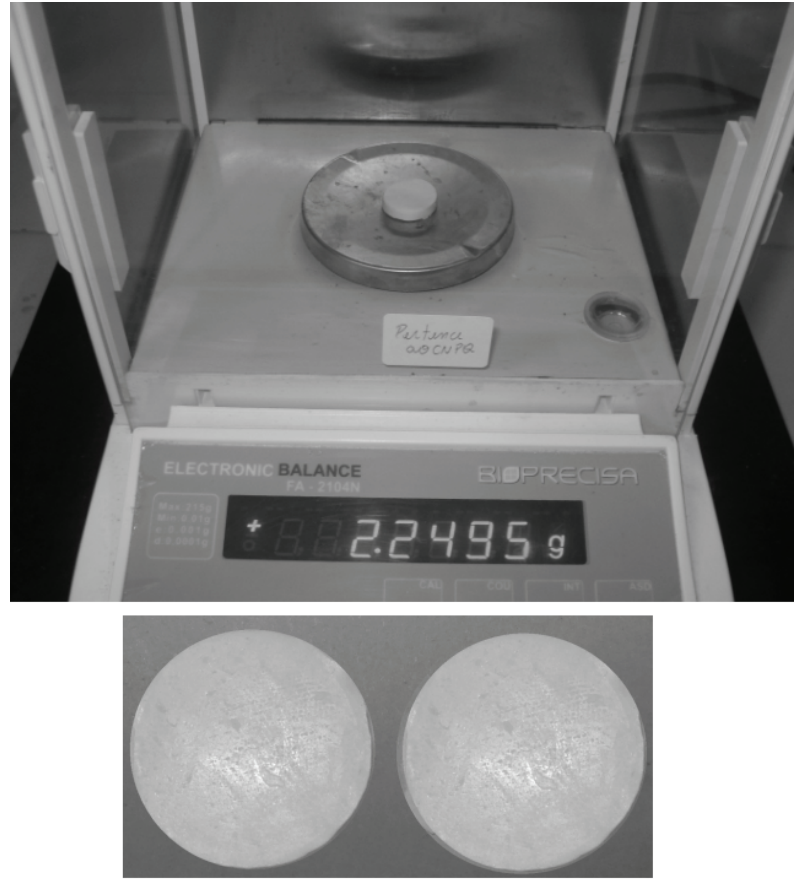

Figura 1 - Tablete de Optosil sendo pesado na balança analítica de precisão para ajuste do peso. Aspecto final ampliado do tablete.

O valor da performance mastigatória de cada voluntário foi calculado através do tamanho médio das partículas trituradas utilizando-se a média geométrica ponderada, pois, as aberturas das peneiras crescem em uma taxa constante, variando de $0,71 \mathrm{~mm}$ até $5,6 \mathrm{~mm}$. A média geométrica ponderada do tamanho das partículas foi calculada por meio da expressão ${ }^{13,14}$ :

$$
\log _{D G M}=\frac{\sum_{i=1}^{k} w_{i} \log _{d_{i}}}{\sum_{i=1}^{k} W_{i}}
$$

Em que:

$\log _{\mathrm{DGM}}=$ logaritmo decimal da média geométrica DGM ponderada do tamanho das partículas, aqui denominada DGM (Diâmetro Geométrico Médio)

$\log _{D I}$ é o logaritmo decimal do diâmetro das peneiras di

com $i=1,2, \ldots, k$ peneiras, ou seja: $\log _{D I}=0,5$ log (diâmetro da primeira peneira em di microns $x$ diâmetro da peneira subsequente em microns);

$W_{1}$ é o peso em gramas das partículas que ficaram i retidas em cada peneira, com $\mathrm{i}=1,2, \ldots, \mathrm{k}$ peneiras.
Assim, o diâmetro geométrico médio (DGM) das partículas foi obtido por meio do antilogaritmo, ou seja:

$$
\mathrm{DGM}=10^{\log }
$$

\section{Aprovação do projeto pelo Comitê de Ética em Pesquisa (CEP)}

O projeto foi aprovado pelo Comitê de Ética em Pesquisa da Universidade Federal de Sergipe, número do protocolo CAAE 0127.0.107.000-09.

\section{Análise estatística}

Os resultados foram tabulados e aplicou-se a análise estatística descritiva para as variáveis estudadas, que consistiram de porcentagem, média e desvio padrão.

Para a comparação da performance mastigatória entre as classes oclusais intragrupo e entre os grupos foram utilizadas ANOVA e Teste T. Para a comparação da performance mastigatória entre os sujeitos respiradores orais e nasais independente da classe oclusal foi utilizado o Teste T. Para todas as análises foi considerado um nível de significância de $5 \%$.

Para as análises foi utilizado o programa GRAPHPAD PRISM (versão 5.0, GraphPad Software. Inc., San Diego, CA, EUA).

\section{RESULTADOS}

Os resultados estão apresentados na seguinte ordem: caracterização dos grupos segundo a faixa etária, gênero e grupo étnico, patologias respiratórios encontradas nos respiradores orais e, por fim estão expostas as comparações da performance mastigatória entre os grupos e intragrupos dependente ou independente da variável oclusão.

Os 60 sujeitos que participaram do estudo foram distribuídos em dois grupos de acordo com o seu padrão respiratório, nasal ou oral. Os grupos $\mathrm{RN}$ e $\mathrm{RO}$ foram constituídos de 30 jovens cada com idade média de 17,87 e 17,83 anos respectivamente. Com relação ao gênero houve uma predominância de sujeitos do gênero feminino no grupo RO e do gênero masculino no grupo $\mathrm{RN}$. Com relação à oclusão morfológica, houve uma distribuição equitativa entre as classes oclusais (Tabela 1 ).

A Figura 2 ilustra a distribuição das patologias respiratórias encontradas no grupo dos respiradores orais. Os cinco diagnósticos mais prevalentes foram hipertrofia de adenóide $(n=12 ; 33,33 \%)$, rinite alérgica $(n=7 ; 19,44 \%)$, sinusite $(n=5 ; 13,88 \%)$, asma $(n=4 ; 11,11 \%)$ e hipertrofia de amígdala $(\mathrm{n}=4 ; 11,11 \%)$. Cada indivíduo pode ter mais de um diagnóstico. 
Tabela 1 - Caracterização da amostra segundo a faixa etária, gênero e classe oclusal

\begin{tabular}{lcc}
\hline Variável/Grupos & Respirador Nasal & Respirador Oral \\
\hline Faixa Etária & & \\
$\quad$ Mínima & 15 & 14 \\
Média & 17,87 & 17,83 \\
Máxima & 22 & 22 \\
$\quad$ Desvio Padrão & 2,2 & 3,1 \\
\hline Gênero & & \\
$\quad$ Masculino & $19(63,3 \%)$ & $13(43,3 \%)$ \\
$\quad$ Feminino & $11(36,7 \%)$ & $17(56,7 \%)$ \\
\hline Classe Oclusal & & \\
Classe I & $10(33,3 \%)$ & $10(33,3 \%)$ \\
Classe II & $10(33,3 \%)$ & $10(33,3 \%)$ \\
Classe III & $10(33,3 \%)$ & $10(33,3 \%)$ \\
\hline
\end{tabular}

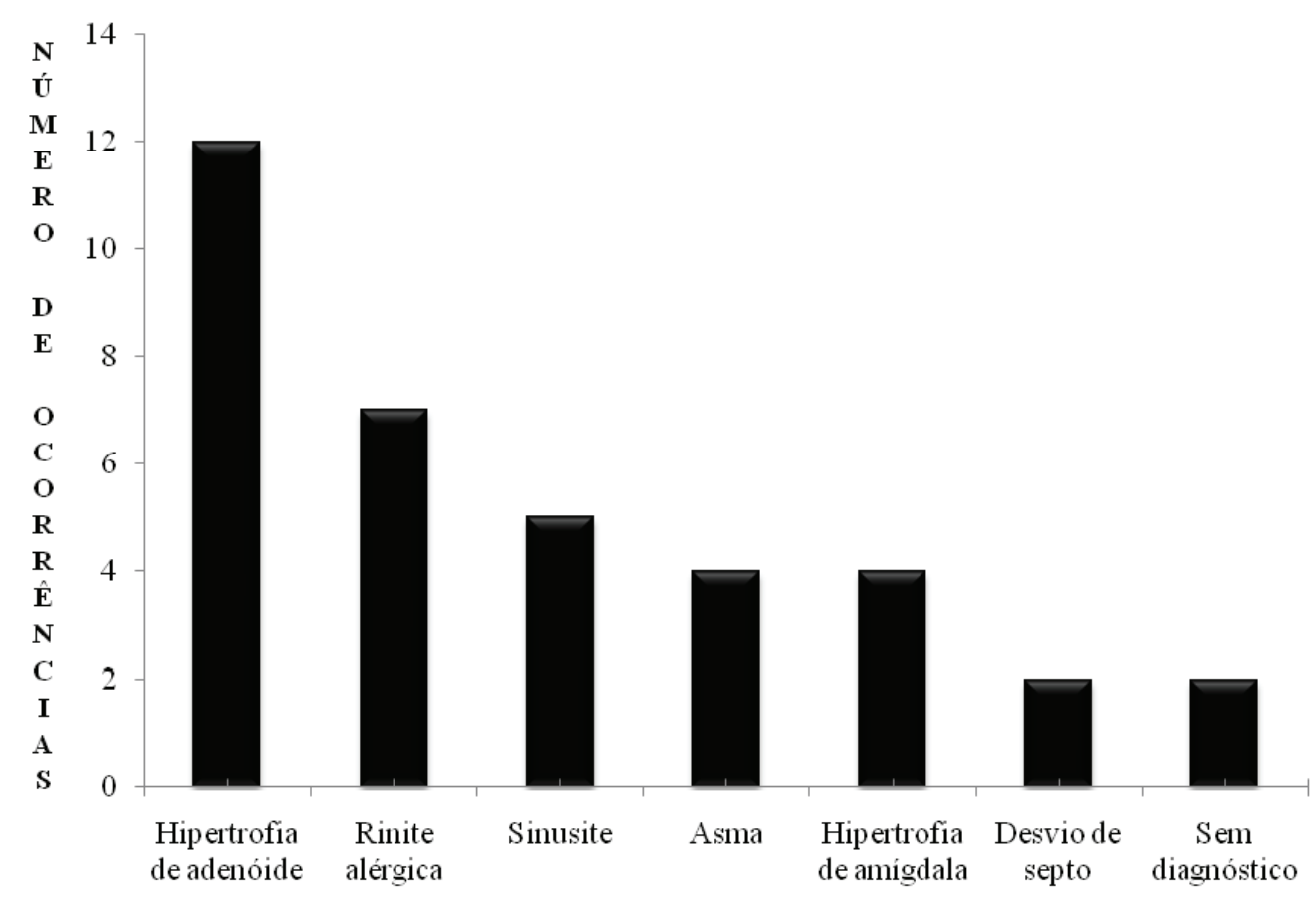

PATOLOGLASRESPIRATÓRIAS

Figura 2 - Patologias respiratórias encontradas no grupo dos respiradores orais

As tabelas 2 e 3 apresentam os valores da performance mastigatória por meio do DGM das partículas mastigadas.

Especificamente na tabela 2 estão apresentados os valores do DGM de acordo com a classe oclusal e padrão respiratório, não sendo verificada diferença significativa do DGM quando comparado as mesmas classes oclusais entre os grupos $\mathrm{RN}$ e $\mathrm{RO}$ (ex: RN-CLI x RO - CLI, etc.) $(p>0,05)$.
Também não foi observada diferença significativa quando a comparação foi entre as diferentes classes oclusais dentro do mesmo grupo (ex: RN-CLI x CLII x CLIII.) ( $p>0,05)$.

Na tabela 3 está exposto o DGM para os grupos $\mathrm{RN}$ e RO, independente da classe oclusal. Também não foi verificada diferença significativa entre a média desses valores entre os grupos $(p>0,05)$. 
Tabela 2 - Diâmetro geométrico médio das partículas mastigadas dos sujeitos com diferentes classes oclusais de acordo com o padrão respiratório

\begin{tabular}{lccc}
\hline & Padrão respiratório & $\mathbf{n}$ & DGM - X \\
\hline \multirow{2}{*}{ Classe I } & Respirador Oral & 10 & 12235 \\
& Respirador Nasal & 10 & 11554 \\
\hline \multirow{2}{*}{ Classe II } & Respirador Oral & 10 & 12420 \\
& Respirador Nasal & 10 & 12855 \\
\hline \multirow{2}{*}{ Classe III } & Respirador Oral & 10 & 12147 \\
& Respirador Nasal & 10 & 11107 \\
\hline
\end{tabular}

Número de indivíduos (N) e Média (X)

Não houve diferença estatística significativa para o DGM entre as mesmas classes oclusais entre os grupos $(p>0,05 ;$ Teste T) e nem entre as diferentes classes oclusais dentro do mesmo grupo ( $p>0,05 ;$ ANOVA)

Tabela 3 - Valor mínimo, máximo e média $(X)$ da performance mastigatória entre os grupos respirador oral e respirador nasal

\begin{tabular}{lcccc}
\hline & $\mathbf{n}$ & MíN & MÁX & X \\
\hline Respirador Oral & 30 & 7601 & 12935 & 12267 \\
Respirador Nasal & 30 & 6776,7 & 12935 & 11839 \\
\hline
\end{tabular}

Número de indivíduos (N), Mínimo (MíN), Máximo (MÁX) e Média (X)

Não houve diferença estatística significativa entre os grupos $R N$ e RO ( $p>0,05$; test T)

\section{DISCUSSÃO}

O objetivo do presente estudo foi comparar a performance mastigatória entre sujeitos com padrões respiratórios nasal e oral associado ou não às características morfológicas da oclusão. Nastri, Bommarito ${ }^{15}$ classificam o padrão respiratório em oral, nasal ou oronasal. Para Silva e colaboradores $^{3}$, o respirador oral é o indivíduo que substitui o padrão correto de respiração nasal por um padrão inadequado, que pode ser oral ou misto. De um modo geral, a respiração exclusivamente oral é rara, havendo na maioria das vezes um padrão misto de respiração oral e nasal nos indivíduos ${ }^{16}$. Dessa forma, alguns autores citam que o uso do termo respirador oral é inapropriado, devendo o mesmo ser substituído por insuficiente respirador nasal ${ }^{17}$. A prevalência da respiração oral na população varia de $5 \%^{18}$ a $55 \%{ }^{19}$.

O diagnóstico do indivíduo respirador oral é essencialmente clínico $^{15}$, não sendo encontrado na literatura um padrão-ouro para esse diagnóstico ${ }^{11}$. No presente estudo optou-se utilizar a placa oronasal para o diagnóstico do padrão respiratório como descrito por Barreto ${ }^{11}$ o qual descreveu a placa oronasal, que é indicada para avaliação do modo respiratório, sendo de utilização simples e acessível a todos os profissionais da saúde por ser de fácil manuseio e propiciar ações intersetoriais e interdisciplinares.

No presente estudo, no grupo dos respiradores nasais, o gênero predominante foi o masculino, com $63,33 \%$. Já no grupo dos respiradores orais, $56,66 \%$ dos participantes eram do gênero feminino. Felcar e colaboradores ${ }^{20}$, também observaram um discreto predomínio de respiração oral no gênero feminino.

Quanto às patologias respiratórias encontradas nos respiradores orais, foi observado que a mais frequente foi a hipertrofia de adenóide $(n=12$; $33,33 \%)$, rinite alérgica $(n=7 ; 19,44 \%)$, sinusite $(n=5$; $13,88 \%)$, asma $(n=4 ; 11,11 \%)$, hipertrofia de amígdala $(n=4 ; 11,11 \%)$ e desvio de septo $(n=2 ; 5,55 \%)$. No estudo de Araújo, Villar, Oliveira, Gomes ${ }^{21}$ a hipertrofia de amígdala, seguida da rinite alérgica, hipertrofia de adenóide e sinusite crônica foram os achados mais frequentes em respiradores orais. Já no estudo de Abreu e colaboradores ${ }^{22}$ a seqüência foi rinite alérgica, hipertrofia de adenóides, hipertrofia de amígdalas e desvio de septo nasal. A asma, que não foi citada nesses estudos, é relacionada à respiração oral em outras pesquisas ${ }^{23,24} \mathrm{Fica}$ evidente, portanto, que embora as prevalências possam variar, hipertrofia de adenóide, de amígdala, rinite alérgica são sempre achados comuns.

Ainda no presente estudo foi comparada a performance mastigatória entre os grupos de respiradores 
nasais e orais associado ou não à característica morfológica da oclusão. Para a análise da performance mastigatória, vários alimentos são descritos na literatura, como os naturais cenoura e amendoim $^{25}$. Entretanto, alguns pesquisadores ${ }^{26,27}$ têm preferido o uso de alimentos artificiais, confeccionados a partir de silicone, isso porque a mesma possui várias vantagens sobre os alimentos naturais, entre elas, ser de fácil mastigação, possuir uma porcentagem alta de deformação, ser facilmente desinfetada, possibilidade de reprodução e padronização dos testes, insolubilidade em água ou em enzimas salivares ${ }^{28}$. Assim, Boretti, Bickel e Geering ${ }^{25}$, afirmam que a mensuração da performance mastigatória é apropriada, desde que seja realizada através de testes laboratoriais e métodos padronizados. Sabe-se, ainda, que o sistema de multi-peneiras (tamises) é considerado um método extremamente viável e vem sendo utilizado desde 1924 para testes de eficiência mastigatória ${ }^{28}$. Portanto, no presente estudo utilizou-se o teste padrão ouro na avaliação da performance mastigatória.

Não foi observada diferença significativa na performance mastigatória entre os grupos $\mathrm{RN}$ e $\mathrm{RO}$ independente do tipo oclusal, entre as mesmas classes oclusais entre os grupos e entre as diferentes classes oclusais dentro do mesmo grupo.

Os achados do presente estudo corroboram com estudos prévios ${ }^{29,30}$ que demonstraram que crianças com diferentes tipos de maloclusões obtiveram performance mastigatória semelhante à de crianças com oclusão considerada normal, independente do tipo de maloclusão apresentada. No entanto, Sánchez-Ayala ${ }^{31}$, reportou que a oclusão altera a performance mastigatória, desde que ele observou em seu estudo que sujeitos com classe III de Angle tiveram uma pior performance mastigatória que sujeitos com classe I.

Uma possível explicação para esses resultados distintos, é que alguns fatores são determinantes na performance mastigatória, tais como a área dos contatos oclusais presentes, a quantidade de excursão lateral durante a mastigação e o número de caninos em função presentes no $\operatorname{arco}^{32-34}$. Ainda, em adultos, a mordida mais forte de esmagamento ocorre nos primeiros molares permanentes ${ }^{35}$ e os participantes do presente estudo, independente do grupo, tinham todos os molares permanentes em oclusão e hígidos o que provavelmente contribuiu para a ausência de diferença na eficiência mastigatória entre as classes oclusais.

Com relação ao padrão respiratório, verifica-se na literatura ${ }^{3,36,37}$ que o padrão respiratório oral pode interferir negativamente na mastigação quanto aos aspectos: tempo mastigatório, sobras de alimento na cavidade oral, postura dos lábios, ruído durante a mastigação e mastigação unilateral. Todavia, Rodrigues e colaboradores ${ }^{38}$, observaram, em seu estudo, que as alterações na função da mastigação não foram estatisticamente significativas quando associado à respiração oral. Assim, podemos observar que o padrão respiratório pode influenciar na mastigação, ou seja, torná-la mais lenta e ruidosa, entretanto, nem sempre a performance mastigatória é alterada pelo padrão respiratório, como observado no presente estudo.

Por fim no presente estudo o fator gênero não foi considerado em termos de comparação da eficiência mastigatória, desde que outros autores relataram que o gênero não influencia no desempenho mastigatório ${ }^{39}$.

Esses resultados em conjunto sugerem que na amostra estudada nem o padrão respiratório oral nem a presença de maloclusão influenciaram negativamente a performance mastigatória em relação aos sujeitos que possuíam padrões respiratório e oclusal adequados. No entanto, estudos futuros são necessários para verificar se a não correção destes distúrbios podem, a longo prazo, influenciar na performance mastigatória.

\section{CONCLUSÃO}

Ao término desse estudo, que teve como objetivo comparar o desempenho mastigatório de indivíduos respiradores nasais e orais na dentição permanente pôde-se averiguar que o tipo de padrão respiratório não foi um fator determinante na performance mastigatória. 


\section{ABSTRACT}

Purpose: the purpose is to evaluate, through the chewing sequence of an Optosil artificial "tablet", the masticatory performance in nasal breathing and mouth breathing. Method: we attended sixty dentate subjects aged 14-22 year old. An Optosil "tablet" was supplied to each participant, who executed 20 masticatory cycles, the Optosil fragments had been placed in a column with seven bolters, and the retained particles were weighed on a precision analytical balance and the masticatory efficiency value was calculated. The gotten data were submitted to the descriptive analysis and inferential analysis (test T and ANOVA). Results: the nasal breathing and mouth breathing groups were composed by 30 young people each, with a mean age of 17.87 and 17.83 year old, respectively. Regarding gender, the females predominated in the mouth breathing group and the males in the nasal breathing group. The five most prevalent diagnoses in the mouth breathing group were adenoid hypertrophy, allergic rhinitis, sinusitis, asthma and tonsillar hypertrophy. Finally, when we carried out the test, there was no statistical difference in masticatory performance in mouth breathing and nasal breathing ( $p>0.05$ ).Conclusion: it was concluded that the breathing pattern was not a determining factor in the masticatory efficiency.

KEYWORDS: Mastication; Efficiency; Mouth Breathing

\section{REFERÊNCIAS}

1. Felício CM, Couto GA, Ferreira CLP, Mestriner JW. Confiabilidade da eficiência mastigatória com beads e correlação com a atividade muscular. Pró-Fono R. Atual. Cient. 2008;20(4):225-30.

2. Santos HL, Santos HL, Santos RP. A importância da visão multidisciplinar no diagnóstico e tratamento de indivíduos com a síndrome do respirador bucal. Rev. ABO Nac. 2008;16(4):230-2.

3. Silva TLP, Held PA, Sampaio LMM, Lorenzo VAPD, Costa D. Síndrome do respirador bucal: uma abordagem fisioterapêutica. Rev. Biocên. 2007;43(1-2):97-104.

4. Paulo CB, Conceição CA. Sintomatologia do respirador oral. Rev CEFAC. 2003;5(3):219-22.

5. Moyers RE. Ortodontia. Rio de Janeiro: Guanabara Koogan; 1991.

6. Juliano ML, Machado MAC, Carvalho LBC, Prado LBF, Prado GF. Mouth breathing children have cephalometric patterns similar to those of adult patients with obstructive sleep apnea syndrome. Arq. Neuro-Psiquiatr. 2009;67(3b):860-5.

7. Lessa FCR, Enoki C, Feres MFN, Valera FCP, Lima WTA, Matsumoto MAN. Breathing mode influence in craniofacial development. Rev. Bras. Otorrinolaringol. 2005; 71(2):156-160.

8. Branco A, Ferrari GF, Weber AS. Alterações orofaciais em doenças alérgicas de vias aéreas. Rev Paul Pediatr. 2007;25(3):266-70.

9. Aragão W. Respirador Bucal. J. Pedriatria. 1988;64(8):349-52.

10. Reis TC, Quaglia C. O adolescente respirador bucal. Adolescência \& Saúde. 2005;3(2):30-2.
11. Barreto, ACYR. Respiração oral proposta de um novo instrumento para avaliação do modo respiratório [tese]. Piracicaba (SP): Universidade Estadual de Campinas; 2003.

12. Araújo MCM. Ortodontia para clínicos. Programa pré-ortodôntico.4. ed. São Paulo:Santos;1988.

13. Spiegel MR. Estatística. 3. ed. São Paulo: Makron Books, p.643, 1993.

14. Prado MMS, Borges TF, Prado CJ, Gomes VL, Neves FD. Função Mastigatória de Indivíduos Reabilitados com Próteses Totais Mucoso Suportadas. Pesq Bras Odontoped Clin Integr. 2006;6(3):259-66.

15. Nastri VHT, Bommarito S. Avaliação da respiração nos diferentes tipos faciais em indivíduos com maloclusão. Revista Odonto. 2007;15(30):97-106.

16. Ribeiro ML. Qualidade de vida no respirador oral: Avaliação sistemática em crianças de 6 a 12 anos, atendidas em centro de referência da UFMG [tese]. Minas Gerais (MG): Faculdade de Medicina - Universidade Federal de Minas Gerais; 2006.

17. Menezes VA, Leal RB, Pessoa RS, Pontes RMES. Prevalência e fatores associados à respiração oral em escolares participantes do projeto Santo Amaro-Recife,2005. Rev Bras Otorrinolaringol. 2006;72(3):394-9.

18. Kharbanda OP et al. Oral habits in school going children of Delhi:a prevalence study. J Indian Soc Pedod Prev Dent. 2003;21(3):120-4.

19. Abreu RR, Rocha RL, Lamounier JA, Guerra AF. Prevalence of mouth breathing among children. J Pediatr (Rio J). 2008;84(5):467-70.

20. Felcar JM, Bueno IR, Massan AC, Torezan RP, Cardoso JR. Prevalence of mouth breathing 
in children from an elementary school. Cien Saude Colet. 2010;15(2):437-44.

21. Araújo CAF, Villar MLGP, Oliveira MR, Gomes MRS. Avaliação etiológica dos respiradores bucais relacionados a idade e sexo no Ambulatório Escola da Faculdade de Medicina de Petrópolis. Revista SORL-RJ. 2009;9(2):91-6.

22. Abreu RR, Rocha RL, Lamounier JA, Guerra AF. Etiology, clinical manifestations and concurrent findings in mouth-breathing children. J Pediatr (Rio J). 2008;84(6):529-35.

23. Campanha SMA, Freire LMS, Fontes MJF. O impacto da asma, da rinite alérgica e da respiração oral na qualidade de vida de crianças e adolescentes. Rev. CEFAC. 2008;10(4):513-9.

24. Menezes VA, Barbosa AMF, Leal RB, Santos JA, Barros LF, Azevedo MFA. Padrão de respiração em crianças asmáticas. Odonto. 2010;18(35):24-9.

25. Boretti G, Bickel M, Geering AH. A review of masticatory ability and efficiency. J Prosthet Dent. 1995;74(4):400-3.

26. Slagter AP. Force-deformation properties of artificial and natural foodas fot testing chewing efficiency. J Posthet Dent. 1992;68(5):790-9.

27. Pocztaruk RL, Frasca LCF, Rivaldo EG, Fernandes EL, Gavião MBD. Protocol for production of a chewable material for masticatory function tests (Optocal - Brazilian version). Braz. oral res. 2008;22(4):305-10.

28. Sierpińska T, Gołębiewska M, Długosz JW. The relationship between masticatory efficiency and the state of dentition at patients with non rehabilitated partial lost of teeth. Advances in Medical Sciences. 2006;51(1):196-9.

29. Lemos AD, Gambareli FR, Serra MD, Pocztaruk $\mathrm{RL}$, Gavião MBD. Chewing performance and bite force in children. Braz J Oral Sci. 2006;5(18):1101-8
30. Toro A, Buschang PH, Throckmorton G, Roldan S. Masticatory performance in children and adolescents with Class I and II malocclusions. Eur J Orthod. 2006;28(2):112-9.

31. Sánchez-Ayala A, Gebert AP, Godoy EP, Martins G, Gomes OMM. A eficiência mastigatória é alterada pela maloclusão? Revista Dens. 2007;15(2):119.

32. Bourdiol P, Mioche L. Correlations between functional and occlusal tooth-surface areas and food texture during natural chewing sequences in humans. Arch Oral Biol. 2000;45:691-9.

33. Kohyama K, Mioche L, Bourdiol P. Influence of age and dental status on chewing behaviour studied by EMG recordings during consumption of various food samples. Gerodontology. 2003;20(1):15-23.

34. Hatch JP, Shinkai RS, Sakai S, Rugh JD, Paunovich ED. Determinants of masticatory performance in dentate adults. Arch Oral Biol. 2001;46(7): 641-8.

35. Mansour RM, Reynick RJ. In vivo occlusal forces and moments. I. Forces measured in terminal hinge position and associated moments. Journal of Dental Research. 1975;54(1):114-20

36. Ferla A, Silva AMT, Corrêa ECR. Electrical Activity of the Anterior Temporal and Masseter Muscles in Mouth and Nasal Breathing Children. Rev. Bras. Otorrinolaringol. 2008;74(4): 588-95.

37. Cunha DA, Silva GAP, Motta MEFA, Lima CR, Silva HJ. A respiração oral em crianças e suas repercussões no estado nutricional. Rev. CEFAC. 2007;9(1):47-54.

38. Rodrigues HOSN, Faria SR, Paula FSG, Motta AR. Ocorrência de respiração oral e alterações miofuncionais orofaciais em sujeitos em tratamento ortodôntico. Rev CEFAC. 2005;7(3):356-62.

39. Helkimo E, Carlsson GE, Helkimo M. Chewing efficiency and state of dentition. A methodologic study. Acta Odonto Scand. 1978;36(1):33-41.

http://dx.doi.org/10.1590/S1516-18462011005000112

RECEBIDO EM: 05/10/2010

ACEITO EM: 01/02/2011

Endereço para correspondência:

Rosany Larissa Brito de Oliveira

Rua José Jacob Dias Pólito, no 396

Inácio Barbosa - Aracaju - SE

CEP: 49040-290

E-mail: rosanylarissa@ hotmail.com 